\title{
STRONG STABILITY IN NONLINEAR PROGRAMMING REVISITED
}

\author{
DIETHARD KLATTE ${ }^{1}$ and BERND KUMMER ${ }^{2}$
}

(Received 24 June 1997; revised 12 December 1997)

\begin{abstract}
The paper revisits characterizations of strong stability and strong regularity of KarushKuhn-Tucker solutions of nonlinear programs with twice differentiable data. We give a unified framework to handle both concepts simultaneously.
\end{abstract}

\section{Introduction}

In this paper, two basic concepts in the perturbation analysis of Karush-KuhnTucker (KKT) points of nonlinear programs are revisited: the strong stability in the sense of Kojima [17] and the strong regularity in the sense of Robinson [29].

We consider nonlinear programs of the type

$$
\mathrm{P}(f, g, h): \quad \min \{f(x) \mid g(x) \leq 0, h(x)=0\},
$$

where $f: \mathbb{R}^{n} \rightarrow \mathbb{R}, g: \mathbb{R}^{n} \rightarrow \mathbb{R}^{m}$ and $h: \mathbb{R}^{n} \rightarrow \mathbb{R}^{r}$ are supposed to be twice continuously differentiable functions. Then the KKT necessary optimality conditions at some primal-dual vector $(x, u, v) \in \mathbb{R}^{n+m+r}$ have the form

$$
\begin{gathered}
D f(x)+D g(x)^{\mathrm{T}} u+D h(x)^{\mathrm{T}} v=0, \\
g(x) \leq 0, \quad u \geq 0, \quad\langle u, g(x)\rangle=0, \quad h(x)=0,
\end{gathered}
$$

where $D f$ denotes the gradient of $f$, and $D g^{\mathrm{T}}\left(D h^{\mathrm{T}}\right)$ is the transpose of the Jacobian matrix of $g(h)$. A point $(x, u, v)$ satisfying (1.2) is said to be a KKT point of $\mathrm{P}(f, g, h)$, and the $x$-part of a KKT point is called a stationary solution of $\mathrm{P}(f, g, h)$.

\footnotetext{
${ }^{1}$ Institut für Operations Research, Universität Zürich, Moussonstr. 15, CH-8044 Zürich, Switzerland

${ }^{2}$ Institut für Mathematik, Humboldt-Universität zu Berlin, Unter den Linden 6, D-10099 Berlin, Germany

(C) Australian Mathematical Society 1999, Serial-fee code 0334-2700/99
} 
The strong stability and regularity notions in this paper refer, roughly speaking, to the following property: given a locally unique solution $z=(x, u, v)$ of (1.2) and some class $\Phi$ of $C^{2}$-perturbations of the data $\varphi=(f, g, h)$, there exists for any small perturbation $\tilde{\varphi} \in \Phi$ a unique KKT point $z(\tilde{\varphi})$ of $\mathrm{P}(\tilde{\varphi})$ near $z$ and the mapping $\tilde{\varphi} \mapsto z(\tilde{\varphi})$ behaves continuously or Lipschitz continuously. For the precise definitions we refer to Section 2 .

Although the theory of strong stability for $C^{2}$ programs was essentially complete already in the late $80 \mathrm{~s}$, several publications of the $90 \mathrm{~s}[2,3,6,7,25,31]$ recover these results by deriving them from the perturbation analysis of more general optimization or variational problems. The purpose of the paper is to recall basic characterizations of strong stability or regularity for nonlinear optimization problems with twice differentiable data. For reason of space restrictions we consider only the case of isolated KKT points, for some extensions we refer to the discussions in the Remarks 2.9 and 3.6 below. Using the approach $[18,19]$ via the regularity of Lipschitzian equations, we give a unified framework to handle strong stability and strong regularity simultaneously. Moreover, we put these results in a historical context, because it seems that some of the basic studies in the 80 s have not been noticed until now by the optimization community.

This paper is dedicated to Bruce Craven and Bertram Mond on the occasion of their retirement.

\section{Strong stability and regularity notions}

In this section, we recall the definitions of strong stability and regularity and report without proofs some interconnections.

Let $\mathrm{P}(f, g, h)$ be as given above. Following Kojima [17] we define a function $F$ with components $F_{1}, F_{2}$ and $F_{3}$ depending on the primal-dual vector $z=(x, y, v) \in \mathbb{R}^{n+m+r}$ such that

$$
\begin{aligned}
& F_{1}(z):=D f(x)+D g(x)^{\mathrm{T}} y^{+}+D h(x)^{\mathrm{T}} v, \\
& F_{2}(z):=g(x)-y^{-} \\
& F_{3}(z):=h(x)
\end{aligned}
$$

where for given $y \in \mathbb{R}^{m}, y^{+}$and $y^{-}$denote the vectors of components $y_{i}^{+}=\max \left\{y_{i}, 0\right\}$ and $y_{i}^{-}=\min \left\{y_{i}, 0\right\}$, respectively. Under the assumptions on $\mathrm{P}(f, g, h), F$ is piecewise differentiable and locally Lipschitzian. $F$ will be called the Kojima function associated with $P(f, g, h)$. Zeros of $F$, also called critical points of $P(f, g, h)$, can be Lipschitzian mapped into KKT points (that is, solutions of (1.2)) and vice versa. 
More precisely, one has

$$
\begin{gathered}
(x, u, v) \mathrm{KKT} \text { point } \Rightarrow(x, u+g(x), v) \text { critical point, } \\
(x, y, v) \text { critical point } \Rightarrow\left(x, y^{+}, v\right) \mathrm{KKT} \text { point. }
\end{gathered}
$$

This will ensure that the stability results may be equivalently formulated both in terms of KKT points and in terms of critical points; in the following we prefer to use the latter notion.

We start by recalling Kojima's [17] notion of a strongly stable stationary solution. Given an open, bounded subset $X$ of $\mathbb{R}^{n}$, we denote by $C^{2}\left(X, \mathbb{R}^{d}\right)$ the linear space of all $C^{2}$ functions from $X$ to $\mathbb{R}^{d}, d=1+m+r$, with zero element $\mathrm{o}$, and define

$$
|\varphi|_{X}:=\sup _{x \in X} \max \left\{\|\varphi(x)\|,\|D \varphi(x)\|, \max _{1 \leq i \leq d}\left\|D^{2} \varphi_{i}(x)\right\|\right\},
$$

where we use the same symbol $\|\cdot\|$ for a given vector norm and its associated matrix norm. Let $B(z, \varepsilon)$ denote the open ball with center $z$ and radius $\varepsilon$ in the underlying space.

DEFINITION 2.1. (Kojima [17].) A stationary solution $\bar{x}$ of $\mathrm{P}(\varphi), \varphi=(f, g, h)$, is said to be strongly stable with respect to $\Phi \subset C^{2}\left(\mathbb{R}^{n}, \mathbb{R}^{d}\right)$, if (i) there are positive constants $\varepsilon$ and $\delta$ such that the problem $\mathrm{P}(\varphi+\tilde{\varphi})$ has a unique stationary solution $x=x(\tilde{\varphi})$ in $B(\bar{x}, \varepsilon)$, whenever $\tilde{\varphi}:=(\tilde{f}, \tilde{g}, \tilde{h}) \in \Phi$ and $|\tilde{\varphi}|_{B(\bar{x}, \varepsilon)}<\delta$ and (ii) the mapping $\tilde{\varphi} \mapsto x(\tilde{\varphi})$ is continuous at o with $\bar{x}=x(0)$.

The next definition (see [16]) is stronger than that of Kojima's [17] original notion of a strongly stable critical point, but it is the appropriate one in the context of isolated critical points which are our concern in the present paper.

DEFINITION 2.2. A critical point $\bar{z}=(\bar{x}, \bar{y}, \bar{v})$ of $\mathrm{P}(\varphi), \varphi=(f, g, h)$, is said to be strongly stable with respect to $\Phi \subset C^{2}\left(\mathbb{R}^{n}, \mathbb{B}^{d}\right)$, if (i) there are positive constants $\varepsilon$ and $\delta$ such that the equation $F(z)+\widetilde{F}(z)=0, z \in B(\bar{z}, \varepsilon)$, has a unique solution $z=z(\tilde{\varphi})$, whenever $\tilde{\varphi}:=(\tilde{f}, \tilde{g}, \tilde{h}) \in \Phi, F+\tilde{F}$ is the Kojima function of $\mathrm{P}(\varphi+\tilde{\varphi})$ and $|\tilde{\varphi}|_{B(\tilde{x}, \varepsilon)}<\delta$ and (ii) the mapping $\tilde{\varphi} \mapsto z(\tilde{\varphi})$ is continuous at o with $\bar{z}=z(0)$. If additionally $\tilde{\varphi} \mapsto z(\tilde{\varphi})$ is Lipschitzian on a $|\cdot|_{B(\tilde{x}, \varepsilon)}$-norm neighborhood of the zero function, then $\bar{z}$ is called strongly L-stable with respect to $\Phi$.

As usual we shall say that a feasible point $x$ of (1.1) satisfies the Linear Independence Constraint Qualification (LICQ) if the system $\left\{D g_{i}(x), i \in I(x), D h_{j}(x), j=\right.$ $1, \ldots, r\}$ is linearly independent, where

$$
I(x):=\left\{i \in\{1, \ldots, m\} \mid g_{i}(x)=0\right\}
$$


is the set of active indices at $x$. It is well-known that if a stationary solution $x$ to (1.1) satisfies LICQ, then the Lagrange multiplier $(y, v)$ in the associated critical point-or, equivalently, $(u, v)$ in $(1.2)$-is unique.

PROPOSITION 2.3. [16, Theorem 2.3]. Suppose that $\Phi \subset C^{2}\left(\mathbb{R}^{n}, \mathbb{R}^{d}\right)$ contains at least all functions $x \mapsto \tilde{\varphi}(x)=\left(a^{r} x, 0,0\right), a \in \mathbb{R}^{n}$. Then the following conditions are equivalent:

(a) $\bar{x}$ is a strongly stable (with respect to $\Phi$ ) stationary solution to $\mathrm{P}(f, g, h), \bar{x}$ satisfies LICQ, and $(\bar{y}, \bar{v})$ is the associated multiplier.

(b) $(\bar{x}, \bar{y}, \bar{v})$ is a strongly stable (with respect to $\Phi$ ) critical point to $\mathrm{P}(f, g, h)$.

The third strong stability notion will be the basic one in the present paper. It was introduced by Clarke [4] in the analysis of Lipschitzian functions and was applied to the Kojima function (respectively, to the KKT system) in [10] for $C^{2}$ problems and in $[19,20]$ for $C^{1.1}$ optimization problems (that is, the involved functions have locally Lipschitzian first derivatives). Put $s:=n+m+r$.

DEFINITION 2.4. A Lipschitzian function $G: \mathbb{R}^{s} \rightarrow \mathbb{R}^{s}$ is said to be regular at $\bar{z}$ if there are positive constants $\varepsilon$ and $\delta$ such that the local inverse mapping $p \mapsto$ $G^{-1}(p) \cap B(\bar{z}, \varepsilon)$ is single-valued and Lipschitzian on $B(0, \delta)$.

In the literature, a function satisfying the previous definition is also called a local Lipschitzian homeomorphism [31,32] or to be Lipschitzian invertible [19]. Although the notion "regularity" is often understood in a weaker sense, we use it here, since in the context of isolated KKT points no confusion will appear. The same definition can be applied to a multifunction $G$. In this sense, it was one of the central concepts in Robinson [29,31] and also in Dontchev and Rockafellar [7]. In our context, we will have $G=F$ and $p=(a, b, c) \in \mathbb{R}^{s}$. Thus the equation $G(\cdot)=p$ just yields the critical points of the perturbed problem

$$
\mathrm{P}(a, b, c): \quad \min \left\{f(x)-a^{\mathrm{T}} x \mid g(x) \leq b, h(x)=c\right\} .
$$

The following proposition is well-known and gives the interrelations between the notions of the Definitions 2.2 and 2.4.

PROPOSITION 2.5. Let $\Phi \subset C^{2}\left(\mathbb{R}^{n}, \mathbb{R}^{d}\right)$ be a perturbation class containing at least all functions $x \mapsto \tilde{\varphi}(x)=\left(x^{T} B x+a^{T} x, b, c\right), B$ a symmetric $(n, n)$-matrix, $a \in \mathbb{R}^{n}$, $b \in \mathbb{R}^{m}, c \in \mathbb{R}^{r}$. Suppose that $\bar{z}$ is a critical point to $\mathrm{P}(f, g, h)$. Then the following conditions are equivalent:

(1) $\bar{z}$ is strongly stable with respect to $\Phi$;

(2) $\bar{z}$ is strongly $L-$ stable with respect to $C^{2}\left(\mathbb{R}^{n}, \mathbb{R}^{d}\right)$; 


\section{(3) F is regular at $\bar{z}$.}

ProOF. Taking into account that, by Proposition 2.3, each of the conditions (1), (2), (3) involves LICQ, the equivalence of (1) and (2)' $\bar{z}$ is strongly stable with respect to $C^{2}\left(\mathbb{R}^{n}, \mathbb{R}^{d}\right)$ has been proved by Kojima [17, Corollary 4.3]. The inclusion $(2)^{\prime} \Rightarrow(3)$ follows from Theorem 3.1 in Kummer [19]. The inclusion (3) $\Rightarrow(2)$ is a consequence of Robinson [31, Lemma 3.1] (or Theorem 2 in [18]) and (2) $\Rightarrow(2)^{\prime}$ is trivial.

Note that the equivalences in the preceding proposition also belong to a set of characterizations of strong stability given in Theorem 4.3 of [16]. It is also worth mentioning that regularity of $F$ at $\bar{z}$ ensures, by Banach's fixed point theorem applied to $z \mapsto F^{-1}(u(z))$, existence, uniqueness and Lipschitz behavior of the solutions $z=z(u)$ to $F(z)=u(z)$ in some $\varepsilon$-neighborhood of $\bar{z}$ if $u: B(\bar{z}, \varepsilon) \rightarrow \mathbb{R}^{d}$ has now a sufficiently small $C^{1}$-norm. In other words, the perturbations of the Kojima function $F$ in Definition 2.2 can be extended to small $C^{1}$ perturbations $F+u$ which are not related to a second optimization problem. With the same result, regular multi-functions can be perturbed. This has been observed, perhaps first, by Robinson [29].

Robinson's [29] way to reformulate the KKT conditions is based on the observation that $z=(x, u, v)$ is a KKT point of $\mathrm{P}(f, g, h)$ if and only if $z$ satisfies the generalized equation

$$
H(z) \in N_{C}(z), \quad z=(x, u, v) \in C,
$$

where $C:=\mathbb{R}^{n} \times \mathbb{R}_{+}^{m} \times \mathbb{R}^{r}, H=\left(H_{1}, H_{2}, H_{3}\right)$ is defined by

$$
\begin{aligned}
& H_{1}(z):=D f(x)+D g(x)^{\mathrm{T}} u+D h(x)^{\mathrm{T}} v \\
& H_{2}(z):=g(x) \\
& H_{3}(z):=h(x)
\end{aligned}
$$

and $N_{C}(z)$ denotes the usual normal cone of $C$ at $z=(x, u, v)$ which has for the above set $C$ the special form

$$
N_{c}(z)=\left\{(0, \eta, 0) \mid \eta_{i}=0 \text { if } u_{i}>0, \eta_{j} \leq 0 \text { if } u_{j}=0\right\}
$$

The natural parameterization of (2.4) leads to

$$
H(z)-p \in N_{C}(z), \quad z \in C
$$

that is, for $p=(a, b, c)$, the solution set of (2.5) describes the critical point set $F^{-1}(a, b, c)$ of $\mathrm{P}(a, b, c)$ up to the transformation between critical points and KKT points. 
Using $f, g, h \in C^{2}$ and linearizing $H_{1}, H_{2}$ and $H_{3}$ at some solution $\bar{z}=(\bar{x}, \bar{u}, \bar{v})$ of (2.4), one has

$$
\begin{aligned}
& L_{1}(z):=H_{1}(\bar{z})+D H_{1}(\bar{z})(z-\bar{z})=D f(\bar{x})+D g(\bar{x})^{\mathrm{T}} u+D h(\bar{x})^{\mathrm{T}} v+Q(x-\bar{x}), \\
& L_{2}(z):=H_{2}(\bar{z})+D H_{2}(\bar{z})(z-\bar{z})=g(\bar{x})+D g(\bar{x})(x-\bar{x}) \\
& L_{3}(z):=H_{3}(\bar{z})+D H_{3}(\bar{z})(z-\bar{z})=h(\bar{x})+D h(\bar{x})(x-\bar{x})
\end{aligned}
$$

where $\mathscr{L}(x, u, v):=f(x)+u^{\mathrm{T}} g(x)+v^{\mathrm{T}} h(x)$ and $Q:=D_{x}^{2} \mathscr{L}(\bar{x}, \bar{u}, \bar{v})$. Put $L=$ $\left(L_{1}, L_{2}, L_{3}\right)$. So one obtains from (2.4) Robinson's [29] linearized system

$$
L(z) \in N_{C}(z), \quad z=(x, u, v) \in C,
$$

which is also solved by $\bar{z}$. The natural parameterization of (2.6) leads to

$$
L(z)-p \in N_{C}(z), \quad z \in C,
$$

where $p=(a, b, c)$. Obviously, (2.7) coincides with the generalized equation (2.5) applied to the parametric quadratic program

$$
\begin{aligned}
\mathrm{PQ}(a, b, c): \min \left\{q(x)-a^{\mathrm{T}} x \mid\right. & g(\bar{x})+D g(\bar{x})(x-\bar{x}) \leq b, \\
h(\bar{x})+D h(\bar{x})(x-\bar{x}) & =c\}
\end{aligned}
$$

where $q(x):=D f(\bar{x})(x-\bar{x})+\frac{1}{2}(x-\bar{x})^{\mathrm{T}} Q(x-\bar{x})$. Now we are on the point of defining Robinson's notion of a strongly stable KKT point, called strong regularity.

DEFINITION 2.6. (Robinson [29]) For some solution $\bar{z}$ of (2.4), the generalized equation (2.4) is said to be strongly regular at $\bar{z}$ if there are positive constants $\varepsilon$ and $\delta$ such that for each $p \in B(0, \delta)$, the parametric linearized system (2.7) has a unique solution $z(p)$ in $B(\bar{z}, \varepsilon)$ and the mapping $p \mapsto z(p)$ is Lipschitzian on $B(0, \delta)$.

It is well-known from the literature that for the standard $C^{2}$ program $\mathrm{P}(f, g, h)$, strong regularity of (2.4) at some KKT point $\bar{z}$ (with related critical point $\tilde{z}$ ) and regularity of the Kojima function $F$ at $\bar{z}$ are equivalent. Since this equivalence will be recovered by our approach in the next section, we shall give the corresponding results and references later.

REMARK 2.7. By Definition 2.4 and Definition 2.6, the generalized equation (2.4) is strongly regular at some KKT point $\bar{z}$ of $\mathrm{P}(f, g, h)$ if and only if the Kojima mapping $F_{P Q}$ associated with the quadratic program $\mathrm{PQ}(0,0,0)$ is regular at $\tilde{z}$, where $\tilde{z}$ is the critical point which is related to $\bar{z}$ by the Lipschitzian transformation (2.2). 
REMARK 2.8. It is easy to see that the strong regularity of (2.4) at a KKT point $\bar{z}=(\bar{x}, \bar{u}, \bar{v})$ implies that $\bar{x}$ satisfies LICQ. Indeed, by construction, the active index sets $I(\bar{x})$ at $\bar{x}$ with respect both to the original problem $\mathrm{P}(f, g, h)$ and to the quadratic approximation PQ $(0,0,0)$ coincide. Now apply Proposition 2.3, Proposition 2.5 and Remark 2.7.

Robinson's main impetus in [29] was the study of inverse and implicit function theorems for generalized equations. This applies to a parametric family of nonlinear programs

$$
\mathrm{P}(t), t \in T: \quad \min _{x}\{f(t, x) \mid g(t, x) \leq 0, h(t, x)=0\}
$$

where $f(t, \cdot), g(t, \cdot), h(t, \cdot), t \in T$, are $C^{2}$ with respect to $x \in \mathbb{R}^{n}$ and all functions, together with the first and second order partial derivatives by $x$, are (at least) continuous in $(t, x)$. By the implicit function theorem [29, Theorem 2.1], strong regularity at a KKT point of the initial problem implies strong stability of the KKT map under small perturbations. Extensions to $C^{1,1}$ programs are studied by Kummer in [20].

REMARK 2.9. (Non-unique multipliers.) Until now we have characterized strong stability of critical points, which involves necessarily LICQ at the primal part. To discuss the case of strong stability of a stationary solution (Definition 2.1 ) in the absence of LICQ, let $\bar{x}$ be a stationary solution to $\mathrm{P}(f, g, h)$ and suppose that $\bar{x}$ satisfies the Mangasarian-Fromovitz Constraint Qualification (MFCQ). Then the set $\Lambda(\bar{x})$ of associated Lagrange multipliers is a bounded convex polyhedron. Kojima [17] has shown the following: If $\bar{x}$ does not satisfy LICQ, then $\bar{x}$ is strongly stable if and only if the strong second-order sufficient optimality condition (SSOC) [17,29] holds, that is, (3.10) below is fulfilled at any point $(\bar{x}, \bar{y}), \bar{y} \in \Lambda(\bar{x})$. In particular, $\bar{x}$ is then an isolated local minimizer.

For the parametric problem $\mathrm{P}(t), t \in T$, this means that MFCQ and SSOC at some stationary solution $\bar{x}$ to $\mathrm{P}(\bar{t})$ imply the existence of a function $t \mapsto x(t)$ continuous in some neighborhood $U$ of $\bar{t}$ such that for $t \in U, x(t)$ is an isolated local minimizer to $\mathrm{P}(t)$ near $\bar{x}=x(\bar{t})$. Supposing that $f, g, h$ are $C^{2}$ with respect to $(x, t)$, Gfrerer [9] has shown that MFCQ and SSOC imply Hölder continuity with order $1 / 2$ of $x(\cdot)$ around $\bar{t}$, while Ralph and Dempe [27] (see also [8,24]) proved that $x(\cdot)$ is even Lipschitzian around $\bar{t}$ if additionally the constant rank condition is satisfied. Without this additional assumption, local Lipschitz continuity may not be expected, however local upper Lipschitz continuity holds, see, for example, Robinson [30]; we refer also to Remark 3.6 below. 


\section{Characterizations of strong stability}

In this section, we characterize simultaneously the strong regularity of the piecewise smooth function $F$ in (2.1) and of the (smooth-polyhedral) generalized equation (2.4) and hence, by Proposition 2.5, the other strong stability notions, too. Our tool is Kummer's approach [19] though one could also use the regularity results in Dontchev and Rockafellar [7] for generalized equations or in Pang and Ralph [26], Ralph and Scholtes [28] for $\mathrm{PC}^{1}$ equations. However, the following approach is applicable also to $C^{1,1}$ optimization problems and Lipschitzian equations, which has not been shown up to now for the other ones. Since we study the stability behavior of the KKT system near a given critical point $\bar{z}=(\bar{x}, \bar{y}, \bar{v})$, equations $h_{i}(x)=0$ may be handled in the same way as inequalities with associated positive multiplier $\bar{y}_{i}$ and so, for simplicity of presentation, we suppose throughout this section that no equations appear. For a $C^{2}$ program without equality constraints,

$$
\mathrm{P}(f, g): \quad \min \{f(x) \mid g(x) \leq 0\},
$$

the Kojima function $F=\left(F_{1}, F_{2}\right)$ according to (2.1) may be written by

$$
F(x, y)=M(x) N(y), M(x):=\left(\begin{array}{ccc}
D f(x) & D g(x)^{\mathrm{T}} & \mathrm{O}_{n, m} \\
g(x) & \mathrm{O}_{m, m} & -E_{m}
\end{array}\right), N(y):=\left(\begin{array}{c}
1 \\
y^{+} \\
y^{-}
\end{array}\right),
$$

where $\mathrm{O}_{n, m}$ is the $(n, m)$ zero matrix and $E_{m}$ is the $(m, m)$ unit matrix. Hence, $F$ is a locally Lipschitzian function of special form: $M(\cdot)$ is continuously differentiable and $N(\cdot)$ is a special piecewise linear function. This suggests to use characterizations of local Lipschitz invertibility of locally Lipschitzian functions by means of generalized directional derivatives, together with suitable chain rules for generalized derivatives.

Given a locally Lipschitzian function $G: \mathbb{R}^{s} \rightarrow \mathbb{R}^{s}$ and a zero $\bar{z}$ of $G$, the kind of derivative we need follows from the characterization of regularity in Kummer [18, 19]:

$$
G \text { is regular at } \bar{z} \Leftrightarrow 0 \notin \Delta G(\bar{z} ; \mu) \forall \mu \in \mathbb{R}^{s} \backslash\{0\},
$$

with $\Delta G(\bar{z} ; \mu)$ being Thibault's [33] so-called limit set which is defined by

$\nu \in \Delta G(\bar{z} ; \mu) \quad: \Leftrightarrow \quad \nu:=\lim _{k} \theta_{k}^{-1}\left(G\left(z^{k}+\theta_{k} \mu\right)-G\left(z^{k}\right)\right)$, where $z^{k} \rightarrow \bar{z}, \theta_{k} \downarrow 0$.

A sufficient condition for regularity was given by Clarke (see, for instance, $[5$, Theorem 7.1.1]): 
where, with $\operatorname{Diff}(G):=\left\{z \in \mathbb{R}^{s} \mid G\right.$ is differentiable at $\left.z\right\}$ and conv $X:=$ convex hull of $X$,

$$
\partial G(\bar{z}):=\operatorname{conv}\left\{\lim _{k} D G\left(z^{k}\right) \mid z^{k} \rightarrow \bar{z}, z^{k} \in \operatorname{Diff} G(k=1,2, \ldots)\right\}
$$

is Clarke's [5, Definition 2.6.1] generalized Jacobian of $G$ at $\bar{z}$. Obviously, Clarke's condition 3.3 is equivalent to $0 \notin \partial G(\bar{z}) \mu$ for all $\mu$ in $\mathbb{R}^{s} \backslash\{0\}$, where $\partial G(\bar{z}) \mu$ is the element-wise product. Let us recall some facts important in our regularity analysis; for the details we refer to [19]. First we note that $\Delta G(\bar{z} ; \mu)$ is a subset of $\partial G(\bar{z}) \mu$ and there are examples that this inclusion may be strict. Moreover, the reverse inclusion of (3.3) is not true, in general. If $G$ is continuously differentiable at $\bar{z}$, then for all $\mu \in \mathbb{R}^{s}, \Delta G(\bar{z} ; \mu)$ and $\partial G(\bar{z}) \mu$ coincide and are equal to $D G(\bar{z}) \mu$.

Coming back to regularity for $C^{2}$ programs, we observe that the situation differs from the general case. Now, identifying $G$ and $F$, Clarke's condition 3.3 is even necessary. This has been proved-in terms of strong stability - by Jongen, Klatte and Tammer [10, Theorem 3.1] and it can be derived from Theorem 1.1 in Kummer [19]. In the following, we shall recover this result and we obtain as by-products also other known characterizations of strong stability or regularity.

Since $M$ is continuously differentiable, $\Delta M(x ; \lambda)$ reduces to the standard directional derivative $M^{\prime}(x ; \lambda)$ and we may apply the chain rule (P8) in [19] to the Kojima function $F(x, y)$ described by (3.1). Hence, one has

$$
\Delta F((x, y) ;(\lambda, \mu))=M^{\prime}(x ; \lambda) N(y)+M(x) \Delta N(y ; \mu) .
$$

Note that, by standard analysis arguments,

$$
M^{\prime}(x ; \lambda)=\left(\begin{array}{ccccc}
D^{2} f(x) \lambda & D^{2} g_{1}(x) \lambda & \cdots & D^{2} g_{m}(x) \lambda & \mathrm{O}_{n, m} \\
D g(x) \lambda & 0 & \cdots & 0 & \mathrm{O}_{m, m}
\end{array}\right)
$$

For completeness, we present the proof of a simple known result, since it is, together with the chain rule (3.4), the key for the characterization theorem.

LEMMA 3.1. [19, Lemma 4.3.] For the function $\Gamma: \mathbb{R} \rightarrow \mathbb{R}^{2}$, defined by $\Gamma(t):=$ $\left(t^{+}, t^{-}\right)$, the following holds: $\Delta \Gamma(0 ; \mu)=\partial \Gamma(0) \mu=\left\{(\alpha \mu,(1-\alpha) \mu)^{T} \mid 0 \leq \alpha \leq 1\right\}$.

PROOF. Trivially, we have $\partial \Gamma(0)=\operatorname{conv}\left\{(1,0)^{\mathrm{T}},(0,1)^{\mathrm{T}}\right\}$. Hence, $D:=\partial \Gamma(0) \mu$ has the claimed representation, and, by the discussion above, it contains $\Delta \Gamma(0 ; \mu)$. Now, let $\nu=\left(\nu_{1}, \nu_{2}\right)^{\mathrm{T}} \in D$ and let $\theta_{k} \downarrow 0$ be given. Then define

$$
t^{k}:= \begin{cases}0, & \text { if } \mu=0, \\ -\theta_{k} \mu \nu_{2}, & \text { if } \mu>0 \\ -\theta_{k} \mu \nu_{1}, & \text { if } \mu<0\end{cases}
$$


Hence $t^{k} \rightarrow 0$ and $\theta_{k}^{-1}\left(\Gamma\left(t^{k}+\theta_{k} \mu\right)-\Gamma\left(t^{k}\right)\right)$ converges to $v$. This implies $D \subset$ $\Delta \Gamma(0 ; \mu)$, which completes the proof.

Given $y \in \mathbb{R}^{m}$, we may divide the vector function $N(\cdot)$ (given according to (3.1)) into components which are differentiable at $y$ or not. Hence, applying Lemma 3.1, we then obtain that $\Delta N(y ; \mu)$ and $\partial N(y) \mu$ coincide and all elements of $\Delta N(y ; \mu)$ have the form

$$
\left(0, \alpha_{1} \mu_{1}, \ldots, \alpha_{m} \mu_{m},\left(1-\alpha_{1}\right) \mu_{1}, \ldots,\left(1-\alpha_{m}\right) \mu_{m}\right)^{\mathrm{T}}, \quad \alpha \in R(y)
$$

where

$$
R(y):=\left\{\begin{array}{l}
\alpha \in \mathbb{R}^{m} \mid \begin{array}{ll}
\alpha_{i}=0, & \text { if } y_{i}<0 \\
\alpha_{i}=1, & \text { if } y_{i}>0 \\
\alpha_{i} \in[0,1], & \text { if } y_{i}=0
\end{array} \quad(i=1, \ldots, m)
\end{array}\right\}
$$

Writing $l(x, y):=f(x)+g(x)^{\mathrm{T}} y^{+}$, we obtain

$$
M^{\prime}(x ; \lambda) N(y)=\left(\begin{array}{c}
D_{x}^{2} l(x, y) \lambda \\
D g(x) \lambda
\end{array}\right) \quad \text { and } \quad M(x) \Delta N(y ; \mu)=\left(\begin{array}{c}
\sum_{i=1}^{r} \alpha_{i} \mu_{i} D g_{i}(x) \\
-\left(1-\alpha_{1}\right) \mu_{1} \\
\vdots \\
-\left(1-\alpha_{m}\right) \mu_{m}
\end{array}\right)
$$

Applying Lemma 3.1, (3.4) and a chain rule for Clarke's generalized Jacobian [5, Theorem 2.6.6], we have for all points $(x, y)$ and directions $(\lambda ; \mu)$ that $\Delta F((x, y),(\lambda, \mu))$ and $\partial F(x, y)\left(\begin{array}{l}\lambda \\ \mu\end{array}\right)$ coincide. This implies a representation of the generalized Jacobian $\partial F(x, y)$, which seems to be new.

LEMMA 3.2. Let $F$ be the Kojima function according to (3.1). Then for $z=(x, y) \in$ $\mathbb{R}^{n+m}, \partial F(z)$ is the set of all matrices $A(z, \alpha), \alpha \in R(y)$, where

$$
A(z, \alpha)=\left(\begin{array}{cccc}
D_{x}^{2} l(x, y) & \alpha_{1} D g_{1}(x) & \cdots & \alpha_{m} D g_{m}(x) \\
D g_{1}(x)^{T} & -\left(1-\alpha_{1}\right) & & \\
\vdots & & \ddots & \\
D g_{m}(x)^{T} & & & -\left(1-\alpha_{m}\right)
\end{array}\right)
$$

By applying (3.2) to the Kojima function (3.1) and its limit set $\Delta F((\bar{x}, \bar{y}) ;(\lambda, \mu))$, the foregoing discussion now immediately gives a characterization theorem which can be considered (in the case of $C^{2}$ programs) as a variant of Theorem 5.1 in [19]. 
THEOREM 3.3. (Characterization Theorem.) Let $\bar{z}=(\bar{x}, \bar{y})$ be a critical point of $\mathrm{P}(f, g)$ and let $F$ be the Kojima function according to (3.1). Then $F$ is regular at $\bar{z}$ if and only if for any $\alpha \in R(\bar{y})$, the system

$$
\begin{aligned}
& D_{x}^{2} l(\bar{x}, \bar{y}) \lambda+\sum_{i=1}^{m} \alpha_{i} \mu_{i} D g_{i}(\bar{x})=0, \\
& D g_{1}(\bar{x})^{T} \lambda-\left(1-\alpha_{1}\right) \mu_{1}=0 \text {, } \\
& D g_{m}(\bar{x})^{T} \lambda \quad-\left(1-\alpha_{m}\right) \mu_{m}=0
\end{aligned}
$$

has only the trivial solution $(\lambda, \mu)=0$.

The foregoing characterization of regularity by system (3.6) is equivalent to the critical face condition in [7, Theorem 5]; for a proof we refer to [21, Lemma 4.2]. By Theorem 3.3 and Lemma 3.2 we immediately have the following equivalence $[10,16,19]$ which was already mentioned above.

COROLLARY 3.4. Let $\bar{z}=(\bar{x}, \bar{y})$ be a critical point of $\mathrm{P}(f, g)$. Then $F$ is regular at $\bar{z}$ if and only if the generalized Jacobian $\partial F(\bar{z})$ is nonsingular.

As a by-product, we obtain once more a fact which also follows from Proposition 2.3 and Proposition 2.5: if $F$ is regular at $\bar{z}=(\bar{x}, \bar{y})$, then $\bar{x}$ satisfies LICQ. To see this by (3.6), one has only to put $\lambda=0, \alpha_{i}=1$ if $\bar{y}_{i} \geq 0$ and $\alpha_{j}=0$ if $\bar{y}_{j}<0$.

Lemma 3.2 and Corollary 3.4 allow a simple proof of Kojima's matrix characterization of strong stability. This will be done here in the equivalent language of regularity of $F$. Let $\operatorname{det} A$ denote the determinant of a matrix $A$. For given $I$ put $\bar{I}:=\{1, \ldots, m\} \backslash I$. Further, we observe that $I(\bar{x})=\left\{i \mid \bar{y}_{i} \geq 0\right\}$.

COROllary 3.5. (Kojima [17, Corollary 4.3].) Let $\bar{z}=(\bar{x}, \bar{y})$ be a critical point of $\mathrm{P}(f, g)$ and put $I^{+}:=\left\{i \mid \bar{y}_{i}>0\right\}$. Then $F$ is regular at $\bar{z}$ if and only iffor all $I$ with $I^{+} \subset I \subset I(\bar{x})$ and all special choices

$$
\alpha_{i}= \begin{cases}1, & \text { if } i \in I, \\ 0, & \text { if } i \in \bar{I},\end{cases}
$$

the numbers $\operatorname{det} A(\bar{z}, \alpha)$ are non-vanishing and have the same sign.

PROOF. The "only if"-direction easily follows from Corollary 3.4 and Lemma 3.2, because any $\alpha$ satisfying (3.7) for $I^{+} \subset I \subset I(\bar{x})=\left\{i \mid \bar{y}_{i} \geq 0\right\}$ belongs to $R(\bar{y})$ and so $\operatorname{det} A(\bar{z}, \alpha)$ is non-vanishing. Since $\partial F(\bar{z})$ is a convex set of nonsingular matrices, the sign of det $A(\bar{z}, \alpha)$ is constant +1 or -1 .

"If"-direction (the method of proof follows [10, Lemma 3.2]): by Corollary 3.4, it suffices to show that if the matrices $A(\bar{z}, \alpha)$ are nonsingular with constant sign $\sigma$ of the 
determinant for any special choice (3.7) with $I^{+} \subset I \subset I(\bar{x})$, then sign $\operatorname{det} A(\bar{z}, \alpha)=$ $\sigma$ for all $\alpha \in R(\bar{y})$.

We may assume that $I^{0}:=\left\{i \mid \bar{y}_{i}=0\right\}$ consists of the last indices $k, \ldots, m$. Only for $i \in I^{0}, \alpha_{i}$ may vary between 0 and 1 , since for $i \notin I^{0}, \alpha_{i}$ is already fixed by the condition $\alpha \in R(\bar{y})$. Now we choose $V:=A\left(\bar{z}, \alpha^{0}\right)$ and $W:=A\left(\bar{z}, \alpha^{1}\right)$ with

$$
\alpha_{i}^{0}:=1, \text { if } i \in\{1, \ldots, m\} \text { and } \alpha_{i}^{\prime}= \begin{cases}1, & \text { if } i \in\{k, \ldots, m-1\}, \\ 0, & \text { if } i=m .\end{cases}
$$

The matrices $V$ and $W$ differ only by the $m$-th column $V_{m}$ and $W_{m}$. Expanding the determinant to this column, one sees that $r(t):=\operatorname{det}(t V+(1-t) W)$ is affine-linear in $t \in \mathbb{R}$. Hence, due to the hypothesis $\operatorname{sign} r(0)=\operatorname{sign} r(1)=\sigma \neq 0$, we obtain sign $r\left(\alpha_{m}\right)=\sigma$ for all $\alpha_{m} \in[0,1]$. Continuing this procedure for any fixed $\alpha_{m} \in[0,1]$ and choices $\alpha^{2}, \alpha^{3}$ of $\alpha$ according to

$$
\alpha_{i}^{2}\left\{\begin{array} { l l } 
{ = 1 , } & { \text { if } i \in \{ k , \ldots , m - 1 \} , } \\
{ = \alpha _ { m } , } & { \text { if } i = m }
\end{array} \quad \text { and } \quad \alpha _ { i } ^ { 3 } \left\{\begin{array}{ll}
=1, & \text { if } i \in\{k, \ldots, m-2\}, \\
=0, & \text { if } i=m-1, \\
=\alpha_{m}, & \text { if } i=m,
\end{array}\right.\right.
$$

and so on, we finally arrive at sign $\operatorname{det} A(\bar{z}, \alpha)=\sigma$ for all $\alpha$ in $R(\bar{y})$. This completes the proof.

REMARK 3.6. (Extensions.) The approach to the conditions in Theorem 3.3 and its corollaries is also suitable for analyzing strong stability of critical points for $C^{1,1}$ programs and for analyzing the local upper Lipschitz continuity of the stationary solution set mapping of $C^{2}$ or $C^{1,1}$ programs (or of more general variational systems). In fact, a transformation of variables allows a reformulation of system (3.6) as a generalized complementarity system, which involves a generalized equation in terms of Thibault's limit set, see [19-21]. Regularity of $F$ means that this generalized complementarity system has only the trivial solution and this is equivalent to the injectivity condition (3.2). Replacing (3.2) by an appropriate injectivity condition, but now in terms of the contingent directional derivative, one obtains a characterization of local upper Lipschitz continuity via a similar generalized complementarity system; see Klatte and Kummer [15]. The difference essentially consists in the different shape of the (generalized) directional derivatives which are used. Note that the result is closely related to a result given by King and Rockafellar [13] and Levy [22] that a map is locally upper Lipschitzian at some point of its graph if and only if its graphical derivative has image $\{0\}$ at 0 ; see also $[8,23]$.

As mentioned above, condition (3.6) in Theorem (3.3) is a special case of the critical face condition for variational inequalities over polyhedral convex sets (see [7]) and 
can be extended to (strong) regularity conditions for generalized Kojima systems (see $[15,21])$. For extensions of stability results to variational inequalities we also refer to [24].

Finally, we note that Kojima's matrix characterization of strong stability (as given in Corollary 3.5) corresponds to the coherent orientation property introduced by Robinson in [32] with respect to normal maps. An extension of Corollary 3.5 says that a normal map is locally a Lipschitzian homeomorphism if and only if this map has coherent orientation; see $[31,32]$ for details.

Now we shall apply the characterization theorem to the case of a strongly regular (in Robinson's sense) critical point. Given a critical point $\bar{z}=(\bar{x}, \bar{y})$ of $\mathrm{P}(f, g), \bar{z}$ is also a critical point of the quadratic program $\operatorname{PQ}(0,0)$, where

$$
\mathrm{PQ}(a, b): \quad \min \left\{q(x)-a^{\mathrm{T}} x \mid \tilde{g}(x) \leq b\right\}, \quad(a, b) \in \mathbb{R}^{n+m},
$$

and $q(x)=D f(\bar{x})(x-\bar{x})+\frac{1}{2}(x-\bar{x})^{\top} D_{x}^{2} l(\bar{x}, \bar{y})(x-\bar{x})$ and $\tilde{g}(x)=g(\bar{x})+D g(\bar{x})(x-$ $\bar{x})$. Note that there is no difference to the problem considered in Section 2, since for the associated KKT point $(\bar{x}, \bar{u})$ (see (2.2)) and the standard Lagrange function $\mathscr{L}(x, u)=f(x)+g(x)^{\mathrm{T}} u$, the matrices $D_{x}^{2} l(\bar{x}, \bar{y})$ and $D_{x}^{2} \mathscr{L}(\bar{x}, \bar{u})$ coincide.

LEMMA 3.7. Let $\bar{z}=(\bar{x}, \bar{y})$ be a critical point of $\mathrm{P}(f, g)$ and denote by $F_{P Q}$ the Kojima function of the quadratic problem $\mathrm{PQ}(0,0)$. Then $\partial F(\bar{z})=\partial F_{P Q}(\bar{z})$.

PROOF. Immediate by definition of $q$ and $\tilde{g}$.

COROLLARY 3.8. Let $\bar{z}=(\bar{x}, \bar{y})$ be a critical point of $\mathrm{P}(f, g)$ and let $\tilde{z}=(\bar{x}, \bar{u})$ be the related KKT point according to (2.2). Then the generalized equation (2.4) is strongly regular at $\tilde{z}$ if and only if the Kojima function $F$ is regular at $\bar{z}$.

PROOF. Apply Lemma 3.7 and Remark 2.7.

We finish this section by specializing the characterization theorem to the case of local minimizers. For a critical point $(\bar{x}, \bar{y})$ of $\mathrm{P}(f, g)$, let $I(\bar{x})$ again be the active index set at $\bar{x}$ and let $I^{+}$be the set of indices $i$ with $\bar{y}_{i}>0$. Define for $I \subset I(\bar{x})$,

$$
W(I):=\left\{\lambda \mid \lambda^{\mathrm{T}} D g_{i}(\bar{x})=0, i \in I\right\} .
$$

The following theorem is well-known, $c f$. the bibliographical note below.

THEOREM 3.9. Let $\bar{z}=(\bar{x}, \bar{y})$ be a critical point of $\mathrm{P}(f, g)$ and suppose that $\bar{x}$ is a local minimizer of this program. Then $F$ is regular at $\bar{z}=(\bar{x}, \bar{y})$ if and only if $\bar{x}$ satisfies LICQ and $D_{x}^{2} l(\bar{x}, \bar{y})$ is positive definite on $W\left(I^{+}\right)$. In this case, if $(x(a, b), y(a, b))$ denotes the critical point of the linearly perturbed program $\mathrm{P}(a, b)$, then $x(a, b)$ is a local minimizer of $\mathrm{P}(a, b)$ whenever $(a, b)$ is sufficiently small. 
PROOF. Before proving the equivalence, we note that the additional proposition is implied by the persistence of both the LICQ and the second-order condition under small perturbations. The proof is straightforward and will be omitted. Another approach to prove the additional proposition was suggested by Kojima [17, Theorem 5.2, Corollary 6.6].

"If"-direction of the equivalence: the concluding Remark 1 in [20] generalizes this assertion to the $C^{1,1}$ case. For completeness, we repeat the arguments. We abbreviate by $S O C$ the second-order condition " $D_{x}^{2} l(\bar{x}, \bar{y})$ is positive definite on $W\left(I^{+}\right)$". Let $F$ be not regular at $\bar{z}$. Then by applying Theorem 3.3, there exist a vector $\alpha \in R(\bar{y})$ and a nontrivial solution $(\lambda, \mu)$ of (3.6). Using $\alpha_{i}=0$ if $\bar{y}_{i}<0$ and the linear independence of $\left\{D g_{i}(\bar{x}), i \in I(\bar{x})\right\}$, we have $\lambda \neq 0$. Then, by definition of $R(\bar{y})$,

$$
\begin{aligned}
& D g_{i}(\bar{x})^{\mathrm{T}} \lambda=0, \text { if } i \in I^{+}, \\
& D g_{i}(\bar{x})^{\mathrm{T}} \lambda=\left(1-\alpha_{i}\right) \mu_{i}, \alpha_{i} \in[0,1], \text { if } i \in I^{0}:=I(\bar{x}) \backslash I^{+} .
\end{aligned}
$$

Applying this and multiplying the first equation in (3.6) on the left by $\lambda^{\mathrm{T}}$, we obtain

$$
\lambda^{\mathrm{T}} D_{x}^{2} l(\bar{x}, \bar{y}) \lambda=-\sum_{i \in I^{0}} \alpha_{i} \mu_{i} \lambda^{\mathrm{T}} D g_{i}(\bar{x})=-\sum_{i \in I^{0}} \alpha_{i}\left(1-\alpha_{i}\right) \mu_{i}^{2} \leq 0 .
$$

Hence, SOC is not satisfied.

"Only if"-direction of the equivalence: suppose that $F$ is regular at $\bar{z}=(\bar{x}, \bar{y})$. Then, by Proposition 2.3 (or by Corollary 3.4), $\bar{x}$ satisfies LICQ with respect to the system $g(x) \leq 0$. Note that, by convention, LICQ is also satisfied if $I(\bar{x})=\emptyset$. SOC will be shown by combining (i) a known perturbation trick (see, for example, $[6,16,17])$ and (ii) an argument (see, for instance, $[12,14]$ ) on the persistence of the local minimizing property under perturbations. Consider

$$
\mathrm{P}(\varepsilon): \quad \min \{f(x) \mid g(x) \leq b(\varepsilon)\}, \varepsilon \geq 0, \quad \text { where } b(\varepsilon)_{i}:= \begin{cases}\varepsilon & \text { if } \bar{y}_{i}=0 \\ 0 & \text { if } \bar{y}_{i} \neq 0 .\end{cases}
$$

Obviously, for any $\varepsilon>0,(\bar{x}, \bar{y})$ is a critical point of $\mathrm{P}(\varepsilon)$, where the active index set of $\bar{x}$ with respect to $g(x) \leq b(\varepsilon)$ coincides with $I^{+}$. It suffices to show that

$$
\exists \varepsilon>0: \quad \bar{x} \text { is a local minimizer of } \mathrm{P}(\varepsilon) .
$$

Indeed if (3.9) holds then

$$
\lambda^{\mathrm{T}} D_{x}^{2} l(\bar{x}, \bar{y}) \lambda \geq 0 \quad \forall \lambda \in W\left(I^{+}\right),
$$

by a classical necessary optimality condition. Theorem 3.6 implies that, in particular, the matrix $A(\bar{z}, \alpha)$ with $\alpha_{i}=0$ if $\bar{y}_{i} \leq 0$ and $\alpha_{i}=1$ if $\bar{y}_{i}>0$ is nonsingular, and so

$$
\left(\begin{array}{cc}
D_{x}^{2} l(\bar{x}, \bar{y}) & D g_{1^{+}}(\bar{x})^{\mathrm{T}} \\
D g_{1^{+}}(\bar{x}) & \mathrm{O}
\end{array}\right)
$$


is also nonsingular, where $g_{1^{+}}$is the vector function built by $g_{i}, i \in I^{+}$. Hence, by a known fact from linear algebra (see, for example, Corollary 2.2 (a) and the references in [11]), we then obtain that the strong inequality in (3.10) is satisfied for $\lambda \in W\left(I^{+}\right) \backslash\{0\}$, that is, SOC holds.

To show (3.9), we first note that, by regularity, $\bar{x}$ is the unique local minimizer of $\mathrm{P}(0)$ in some closed $\delta$-neighborhood of $\bar{x}$. Then, the continuity of $f, g$, together with LICQ, imply that the mapping $\varepsilon \mapsto S(\varepsilon)$ is upper semi-continuous at 0 , with $S(0)=\{\bar{x}\}$, where $S(\varepsilon):=\operatorname{argmin}\{f(x) \mid g(x) \leq b(\varepsilon),\|x-\bar{x}\| \leq \delta\}$. For the definitions and arguments in detail we refer to [1, Chapter 4]. Thus, for any $\varrho>0$, there is some $\varepsilon^{\prime}>0$ such that for $0<\varepsilon<\varepsilon^{\prime}$, each element of $S(\varepsilon)$ belongs to the interior of $B(\bar{x}, \varrho)$ and is hence a local minimizer of $\mathrm{P}(\varepsilon)$. Since LICQ persists under small perturbations, $\varepsilon$ can be chosen small enough that each element of $S(\varepsilon)$ is a stationary solution of $\mathrm{P}(\varepsilon)$. From the regularity of $F$ at $\bar{z}=(\bar{x}, \bar{y})$ (which is also a critical point of $\mathrm{P}(\varepsilon)$ for positive $\varepsilon$ ) we know that for some small $\varepsilon, \varrho>0, \bar{z}$ is the unique critical point of $\mathrm{P}(\varepsilon)$ in $B(\bar{z}, \varrho)$, and so, $S(\varepsilon)=\{\bar{x}\}$ if $\varepsilon$ is sufficiently small. Therefore, (3.9) is shown, which completes the proof.

Bibliographical Note. The pioneering papers by Kojima [17] and Robinson [29], both published in 1980, already covered many basic characterizations and consequences of strong stability (regularity). In [17, Theorem 3.3, Corollary 4.3], it was proved that the linearization of the Kojima function $F$ is one-to-one if and only if the matrix condition of Corollary 3.5 above is satisfied and that this is equivalent to the strong stability of the corresponding stationary solution $\bar{x}$, provided that LICQ holds. Robinson [29, Theorem $3.1, \S 4$ ] gave a matrix characterization of strong regularity at some critical point $\bar{z}$ by defining a partition of the matrix $A(\bar{z},(1, \ldots, 1))$ into blocks corresponding to the equality(-like) constraints, the inactive inequality constraints and the rest, and by using the Schur complement concept. Additionally supposing LICQ, Jongen et al. [11] proved in 1987 that Robinson's and Kojima's matrix conditions are equivalent, and so, in principle, the equivalence of strong regularity and strong stability is known since that time. A little gap was remaining after the mentioned papers: the proof that LICQ is a (simple!) consequence of regularity, strong regularity and strong stability at critical points. In our knowledge this gap was first closed by [16, Theorem 2.3], [18, Theorem 4], [19, Theorem 5.1].

As mentioned in the proof of Proposition 2.5, the interrelations between all strong stability notions considered in the present paper are intensively studied in $[16,19]$.

The "if"-part of Theorem 3.9 in terms of strong regularity at critical points was proved in [29, Theorem 4.1]. Under the additional assumption of LICQ, Theorem 3.9 was proved in [17, Corollary 6.6] in terms of a strongly stable local minimizer. Since LICQ is automatically satisfied at a strongly stable critical point (all the more if the $x$-part is a local minimizer), Theorem 3.9 should be attributed to Kojima. A 
direct proof of the "only if"-direction of Theorem 3.9 has been recently proposed by Dontchev [6], however, presupposing that in the KKT points $(x(a, b), y(a, b))$ of the linearly perturbed problem, $x(a, b)$ are local minimizers. Applying stability results for non-smooth equations or variational inequalities, proofs of Theorem 3.9 are also given in $[3,7,19,20,25]$.

\section{Acknowledgment}

The authors thank an anonymous referee for his constructive criticism. This work was supported by the Deutsche Forschungsgemeinschaft.

\section{References}

[1] B. Bank, J. Guddat, D. Klatte B. Kummer and K. Tammer, Non-Linear Parametric Optimization (Akademie, Berlin, 1982).

[2] F. Bonnans and A. Shapiro, "Optimization problems with perturbations, a guided tour", SIAM Review 40 (1998) 228-264.

[3] F. Bonnans and A. Sulem, "Pseudopower expansion of solutions of generalized equations and constrained optimization problems", Math. Programming 70 (1995) 123-148.

[4] F. Clarke, "On the inverse function theorem", Pacific J. Math. 64 (1976) 97-102.

[5] F. Clarke, Optimization and Nonsmooth Analysis (Wiley, New York, 1983).

[6] A. Dontchev, "A proof of the necessity of LI and SSOSC for Lipschitzian stability in nonlinear programming", preprint Math. Reviews, Ann Arbor, M148104, USA, May 1997.

[7] A. Dontchev and R. T. Rockafellar, "Characterizations of strong regularity for variational inequalities over polyhedral convex sets", SIAM J. Optimization 6 (1996) 1087-1105.

[8] A. Dontchev and R. T. Rockafellar, "Characterizations of Lipschitz stability in nonlinear programming”, in: Mathematical Programming with Data Perturbations (ed. A. V. Fiacco), (Marcel Dekker, New York, 1997) 65-82.

[9] H. Gfrerer, "Hölder continuity of solutions of perturbed optimization problems under MangasarianFromovitz Constraint Qualification", in: Parametric Optimization and Related Topics (eds. B. Kummer, J. Guddat, H. Th. Jongen and F. Nožička), (Akademie, Berlin, 1987) 113-124.

[10] H. Th. Jongen, D. Klatte and K. Tammer, "Implicit functions and sensitivity of stationary points", Math. Programming 49 (1990) 123-138.

[11] H. Th. Jongen, T. Möbert, J. Rückmann and K. Tammer, "On inertia and Schur complement in optimization", Linear Algebra Appl. 95 (1987) 97-109.

[12] H. Th. Jongen, T. Möbert and K. Tammer, "On iterated minimization in nonconvex optimization", Math. Oper. Res. 11 (1986) 679-691.

[13] A. King and R. T. Rockafellar, "Sensitivity analysis for nonsmooth generalized equations", Math. Programming 55 (1992) 341-364.

[14] D. Klatte and B. Kummer, "Stability properties of infima and optimal solutions of parametric optimization problems", in: Nondifferentiable Optimization: Motivations and Applications (eds. V. F. Demyanov and D. Pallaschke), (Springer, Berlin, 1985) 215-229. 
[15] D. Klatte and B. Kummer, "Generalized Kojima-functions and Lipschitz stability of critical points", manuscript, Institut Operations Research, Universität Zürich, and Institut für Mathematik, Humboldt-Universität, Berlin, December 1997.

[16] D. Klatte and K. Tammer, "Strong stability of stationary solutions and Karush-Kuhn-Tucker points in nonlinear optimization", Ann. Oper. Res. 27 (1990) 285-308.

[17] M. Kojima, "Strongly stable stationary solutions on nonlinear programs", in: Analysis and Computation of Fixed Points (ed. S. M. Robinson), (Academic Press, New York, 1980) 93-138.

[18] B. Kummer, "The inverse of a Lipschitz function in $\mathbb{R}^{n}$ : Complete characterization by directional derivatives", Preprint No. 195, (Humboldt-Universität, Sektion Mathematik, Berlin, 1988).

[19] B. Kummer, "Lipschitzian inverse functions, directional derivatives and application in $C^{1.1}$ optimization", J. Optim. Theory Appl. 70 (1991) 559-580.

[20] B. Kummer, "An implicit function theorem for $C^{0,1}$-equations and parametric $C^{1,1}$-optimization", J. Math. Analysis Appl. 158 (1991) 35-46.

[21] B. Kummer, "Lipschitzian and pseudo-Lipschitzian inverse functions and applications to nonlinear programming", in: Mathematical Programming with Data Perturbations (ed. A. V. Fiacco), (Marcel Dekker, New York, 1997) 201-222.

[22] A. B. Levy, "Implicit multifunction theorems for the sensitivity analysis of variational conditions", Math. Programming 74 (1996) 333-350.

[23] A. B. Levy and R. T. Rockafellar, "Sensitivity of solutions in nonlinear programs with nonunique multiplier", in: Recent Advances in Nonsmooth Optimzation (eds. D. Z. Du, L. Qi and R. S. Womersley), (World Scientific Press, 1995) 215-223.

[24] J. Liu, “Strong stability in variational inequalities”, SIAM J. Control Optim. 33 (1995) 725-749.

[25] J. -S. Pang, "Necessary and sufficient conditions for solution stability of parametric nonsmooth equations", in: Recent Advances in Nonsmooth Optimization (eds. D. Z. Du, L. Qi and R. S. Womersley), (World Scientific Press, 1995) 261-288.

[26] J. -S. Pang and D. Ralph, "Piecewise smoothness, local invertibility, and parametric analysis of normal maps", Math. Oper. Res. 21 (1996) 401-426.

[27] D. Ralph and S. Dempe, "Directional derivatives of the solution of a parametric nonlinear program", Math. Programming 70 (1995) 159-172.

[28] D. Ralph and S. Scholtes, "Sensitivity analysis of composite piecewise smooth equations", Math. Programming B 76 (1997) 593-612.

[29] S. M. Robinson, "Strongly regular generalized equations", Math. Oper. Res. 5 (1980) 43-62.

[30] S. M. Robinson, "Generalized equations and their solutions, Part II: Applications to nonlinear programming”, Math. Programming Study 19 (1982) 200-221.

[31] S. M. Robinson, "An implicit function theorem for a class of nonsmooth functions", Math. Oper. Res. 16 (1991) 292-309.

[32] S. M. Robinson, "Normal maps induced by linear transformations", Math. Oper. Res. 17 (1992) 691-714.

[33] L. Thibault, "On generalized differentials and subdifferentials of Lipschitz vector-valued functions", Nonlinear Anal.: Theory, Methods, and Appl. 6 (1982) 1037-1053. 